\title{
Trouillon, Jean-Louis. Approches de l'anglais de spécialité
}

Perpignan : Presses Universitaires de Perpignan, 2010

\section{Marie-Françoise Narcy-Combes}

\section{CpenEdition}

\section{Journals}

Édition électronique

URL : https://journals.openedition.org/asp/2481

DOI : 10.4000/asp.2481

ISSN : 2108-6354

\section{Éditeur}

Groupe d'étude et de recherche en anglais de spécialité

Édition imprimée

Date de publication : 1 novembre 2011

Pagination : 125-126

ISSN : 1246-8185

\section{Référence électronique}

Marie-Françoise Narcy-Combes, «Trouillon, Jean-Louis. Approches de l'anglais de spécialité », ASp [En ligne], 60 | 2011, mis en ligne le 10 novembre 2011, consulté le 07 décembre 2022. URL : http:// journals.openedition.org/asp/2481; DOI : https://doi.org/10.4000/asp.2481

Ce document a été généré automatiquement le 2 novembre 2020.

Tous droits réservés 


\section{Trouillon, Jean-Louis. Approches de l'anglais de spécialité}

Perpignan : Presses Universitaires de Perpignan, 2010

Marie-Françoise Narcy-Combes

\section{RÉFÉRENCE}

Trouillon, Jean-Louis. 2010. Approches de l'anglais de spécialité. Perpignan, France :

Presses Universitaires de Perpignan, 292 p. ISBN 978-2-35412-073-3. 
1 La parution de l'ouvrage de Jean-Louis Trouillon, maître de conférences HDR à l'Université de Perpignan Via Domitia, intitulé Approches de l'anglais de spécialité, vient occuper un créneau qui paradoxalement avait été laissé libre jusque-là : celui d'une approche didactique de l'anglais de spécialité (ASP) à l'intention des futurs praticiens. JeanLouis Trouillon s'est intéressé durant toute sa carrière à l'enseignement et à la recherche en ASP et c'est en spécialiste qu'il nous offre avec cet ouvrage un autre regard sur ce domaine déjà bien exploré. L'ouvrage comprend sept chapitres. Le premier ouvre le livre sur un aperçu théorique à travers lequel l'auteur s'efforce de cerner la notion de langue de spécialité, tandis que le dernier le clôt sur des conseils pratiques pour ceux qui se

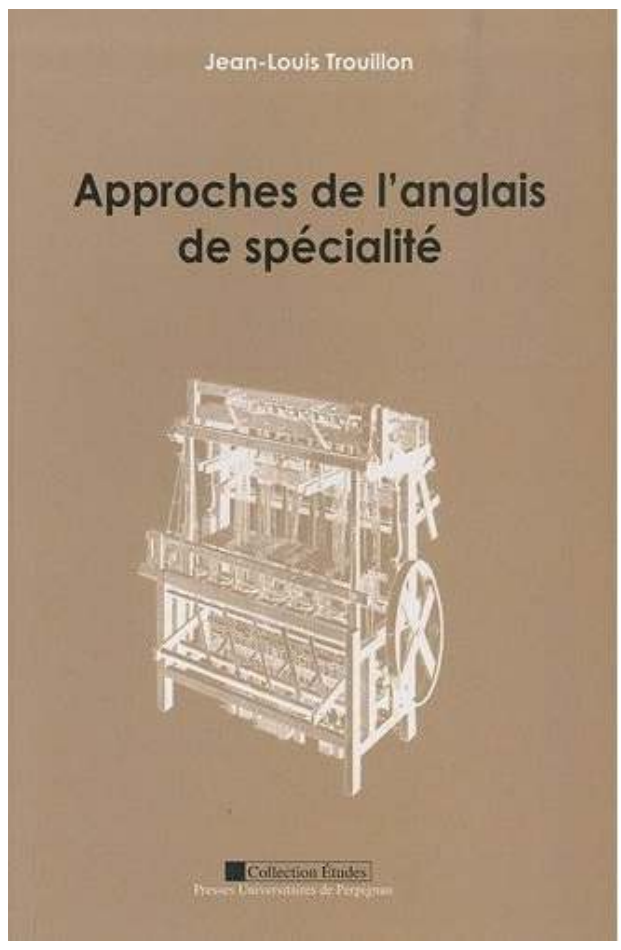
destinent à l'enseignement de l'ASP. Ce parcours de la théorie à la pratique est jalonné de cinq chapitres ancrés dans l'expérience professionnelle de l'auteur dont il tire des exemples précis pour illustrer son propos : anglais du droit (chapitre 2), des sciences (chapitre 3), des forces armées (chapitre 4), du sport (chapitre 5) et du tourisme (chapitre 6). Cet ancrage permet à l'auteur de multiplier les angles d'approche de son sujet: construction de l'objet (chapitre 2), l'anglais comme langue véhiculaire (chapitre 3), les interactions entre langue et culture (chapitre 4), les notions de registre et de genre (chapitre 5) et la place de l'enseignement institutionnel de l'anglais (chapitre 6).

2 L'objectif de l'ouvrage, clairement indiqué dans la préface, est de proposer aux étudiants qui se destinent à l'enseignement de l'anglais, ainsi qu'aux enseignants qui souhaiteraient s'y engager, d'explorer le domaine de la langue de spécialité. L'auteur part en effet du constat que nombre de postes offerts aux concours sont liés à ce type d'enseignement, et force est de constater que cet aspect est encore aujourd'hui largement négligé, comme s'il allait de soi que les praticiens néophytes sauraient faire face à la situation le moment venu. La lecture de cet ouvrage suffit à se convaincre qu'il n'en est rien et que l'enseignement dans les filières professionnalisantes ne s'improvise pas plus qu'ailleurs. En effet, informé, étayé de nombreuses citations et références (plus de 150 références bibliographiques ${ }^{1}$ ), l'ouvrage est bien loin de cautionner la thèse de l'improvisation créatrice: c'est avec du travail, des connaissances solides dans les domaines de spécialité auxquels il s'intéresse et une méthodologie rigoureuse que l'enseignant d'ASP pourra faire face à la tâche qu'il aura à accomplir. En ce sens, et comme l'auteur le souligne lui-même, cet ouvrage peut également guider les premiers pas de jeunes chercheurs qui souhaiteraient trouver là un objet d'étude.

3 L'ouvrage est rédigé dans une langue limpide et il se lit agréablement, ce qui révèle des qualités de pédagogue qui sait accrocher son auditoire dans les premières pages et ne plus le lâcher. Pas de jargon obscur de nature à décourager le public que cible ce livre, 
mais un style direct et accessible, dont Karl Popper nous rappelle qu'il est l'apanage des érudits véritables. L'auteur conduit pas à pas son lecteur dans la compréhension des spécificités de l'anglais de spécialité, ce qui devrait lui permettre de construire le cadre pour élaborer à son tour un programme d'enseignement et/ou de recherche dans la spécialité choisie. On peut regretter cependant que les conclusions en fin de chapitre ne comprennent pas une synthèse des points importants à retenir pour le lecteur, comme un coup de projecteur de nature à renforcer le propos. Les fins de chapitres ajoutent au contraire des informations complémentaires au chapitre, ce qui crée une confusion, car le lecteur pressé ne peut retrouver facilement les idées exprimées au hasard de chaque conclusion qui toutes mériteraient de pouvoir faire l'objet d'une identification rapide sous un intitulé explicite.

4 L'enseignant ou le futur enseignant d'anglais de spécialité trouvera donc dans cet ouvrage une mine d'illustrations de ce qu'est l'ASP. L'ouvrage traite avec beaucoup de clarté des problèmes de terminologie et de types de discours lié à l'enseignement de la langue de spécialité ; en revanche, il n'aborde pas le point de vue de l'apprenant et ne s'interroge pas sur la conception de tâches qui puissent permettre l'appropriation par ce dernier des concepts exposés. Ces aspects pourraient sans doute faire l'objet d'un prochain ouvrage.

\section{NOTES}

1. Sur ce point, on note que l'ouvrage rend hommage à la communauté universitaire en France dont les recherches portent sur l'anglais dans le secteur LANSAD.

\section{AUTEURS}

\section{MARIE-FRANÇOISE NARCY-COMBES}

Université de Nantes 